\title{
Relationship of Peak Expiratory Flow Rate with Waist Circumference, Hip Circumference, and Waist-to-Hip Ratio in Young Adults
}

\author{
Richa Hirendra Rai $\quad$ Shailly Gupta ${ }^{2} \quad$ Asif Mohd² \\ ${ }^{1}$ School of Physiotherapy, Delhi Pharmaceutical Sciences and \\ Research University, New Delhi, India \\ Address for correspondence Shailly Gupta, BPT, 632/43, Joor Bagh, \\ Tri Nagar, Delhi 110035, India (e-mail: shaillygupta9@gmail.com).
}

2Banarsidas Chandiwala Institute of Physiotherapy, New Delhi, India

Ann Natl Acad Med Sci (India) 2020;56:26-29

\begin{abstract}
Introduction It has been observed that respiratory problems and the consequent pulmonary dysfunction are associated with an increase in waist circumference (WC), a measure of obesity. Both comorbidities result in changes in the overall health, quality of life, and life span. The literature shows that body fat distribution can affect pulmonary function. Pulmonary function test (PFT) is a basic and essential tool for the diagnosis and assessment of pulmonary dysfunction, lung diseases, and treatment effects. However, the facilities to measure PFT are not readily available for individual use. Peak expiratory flow rate (PEFR) is a simple and important biomarker that can measure the pulmonary function and can help monitor lung function to initiate early preventive measure. PEFR can be measured by a portable device, which can be carried anytime anywhere. Using this portable instrument, this study was undertaken to investigate whether there is any relationship of PEFR with the biomarkers of obesity, such as WC, hip circumference (HC), and waist-to-hip ratio (WHR) in young adults.

Materials and Methods A total of 84 participants of either sex (38 males and 46 females) aged 20 to 26 years were recruited from a college of South Delhi. Demographic and anthropometric data regarding WC, HC, and WHR were recorded. For PEFR, three consecutive measurements were taken at about 2-minute intervals, and the maximum of the three values was taken into account.

Keywords

- PEFR

- waist circumference

- hip circumference

- waist-to-hip ratio

- obesity

- lung function

Results Though not statistically significant, but a tendency of negative correlation was observed between PEFR and WC $(r=-0.43)$ and between PEFR and WHR $(r=-0.48)$ in males. However, no significant correlation of PEFR with any of the obesity markers was found in females. No correlation was observed between PEFR and HC in the participants of either sex.

Conclusion PEFR may be adversely affected with increase in WC and WHR, as seen in males. However, no association was observed between PEFR and $\mathrm{HC}$ in participants of either sex.
\end{abstract}

\section{Introduction}

With the adoption of western culture, urbanization, and sedentary lifestyle, obesity has become one of the major health problems and a cardinal issue in most of the population in India. The World Health Organization defines obesity as "a condition with excessive fat accumulation in the body to the extent that the health and wellbeing are adversely affected." Obesity results from a complex interaction of genetic, behavioral, environmental, cultural, and
DOI https://doi.org/ 10.1055/s-0040-1712341 ISSN 0379-038X.
License terms

() (1) $\odot \circledast$ 
socioeconomic confounding factors causing an imbalance in energy production and expenditure. Weight and body mass index (BMI) are used as the measures of overall adiposity, whereas waist circumference (WC), hip circumference (HC), and waist-to-hip ratio (WHR) are used as the measures of abdominal obesity and to indicate adverse outcomes of cardiovascular morbidity. ${ }^{1,2}$

It has been observed that an increase in WC is commonly associated with respiratory problems and consequent pulmonary dysfunctions. Both comorbidities then lead to changes in the overall health, quality of life, and life span.,3

Pulmonary function test (PFT) is a basic and essential tool for the diagnosis and assessment of lung dysfunction, diseases, and treatment effects. Peak expiratory flow rate (PEFR) is the maximum flow rate generated during a forceful exhalation, starting from full lung inflation. ${ }^{5}$ It is the maximal airflow during the effort dependent portion of the expiratory maneuver and reflects the large airway flow and depends on the voluntary effort and muscular strength of the participant. The ease with which one can measure PEFR with the help of a portable device can be beneficial for short- and long-term monitoring and can provide the health professionals with a reliable and objective measurement for early detection and therapeutic intervention. ${ }^{6}$ This study was undertaken to investigate whether there is any relationship of PEFR with the biomarkers of obesity, such as WC, HC, and WHR in young adults.

\section{Materials and Methods}

A total of 84 participants of either sex (38 males and 46 females) aged 20 to 26 years from a college of South Delhi who were capable of understanding the procedure and consented to participate were recruited for the study. Participants having a history of current infection, recent hospitalization, cardiopulmonary problems, or musculoskeletal, systemic, and neuropsychological conditions that could affect the outcome of the study were excluded.

\section{Procedure}

After noting the demographic details of the participant, the WC was measured in $\mathrm{cm}$ at a point midway between the lower rib and iliac crest using nonstretchable flexible tape in a horizontal plane at the end of normal expiration, with the participant standing erect with feet together and looking straight forward. The HC was measured in centimeters at the widest girth of the hip. ${ }^{7}$ Using Wright's peak flow meter, PEFR was measured after applying a nose clip on the participant's nose, who was then instructed to take maximum inspiration and blow into the mouthpiece as rapidly, forcefully and completely as possible. Participants were trained well to blow into the instrument maintaining a tight seal between the lips and mouthpiece of the peak flow meter, before starting the actual measurement maneuver. Three attempts were made consecutively by the participants at about 2-minute intervals, and the highest of the three values (L/second) was recorded. After the procedure, the participant was thanked for his/her effort and participation.

Statistical analysis of the data was performed using SPSS Version 20.0 for Windows (IBM Corp., Armonk, New York, United States). A two-tailed $(\alpha=2) p$-value of $<0.05$ was considered to be the level of significance.

\section{Results}

The demographic and anthropometric data and PEFR were analyzed for Pearson's correlation coefficient, and the values of each parameter are presented in -Tables $\mathbf{1}$ and $\mathbf{2}$. - Table 1 shows a summary of the anthropometric and PEFR measurements of the studied participants. - Table 2 shows the analysis of combined data for both the genders and reveals that there is an overall positive correlation between PEFR and WC $(r=0.22)$ as well as between PEFR and WHR $(r=0.37)$, but it was not statistically significant $(p>0.05)$. A negative correlation was observed between PEFR and WC $(r=-0.43)$ and between PEFR and WHR $(r=-0.48)$ in males. Though the results are not statistically significant, there is a tendency of negative correlation between PEFR and WC as well as between PEFR and WHR in male participants. However, no significant correlation of PEFR with any of the

Table 1 Demographic and anthropometric data and PEFR of participants

\begin{tabular}{|l|l|l|}
\hline Parameters, mean \pm SD & Male, $\boldsymbol{n}=\mathbf{3 8}$ & Female, $\boldsymbol{n}=\mathbf{4 6}$ \\
\hline Age $($ years $)$ & $20.44 \pm 1.41$ & $21.42 \pm 1.57$ \\
\hline Height $(\mathrm{cm})$ & $172 \pm 5.70$ & $157 \pm 5.32$ \\
\hline Weight $(\mathrm{kg})$ & $67.12 \pm 9.87$ & $53.15 \pm 9.95$ \\
\hline BMI $\left(\mathrm{kg} / \mathrm{m}^{2}\right)$ & $22.65 \pm 2.91$ & $21.46 \pm 3.63$ \\
\hline Waist circumference $(\mathrm{cm})$ & $80.36 \pm 7.52$ & $72.93 \pm 9.96$ \\
\hline Hip circumference $(\mathrm{cm})$ & $93.81 \pm 6.50$ & $93.47 \pm 7.60$ \\
\hline Waist-to-hip ratio & $0.85 \pm 0.04$ & $0.77 \pm 0.05$ \\
\hline PEFR (L/second) & $455 \pm 91.65$ & $264 \pm 51.85$ \\
\hline
\end{tabular}

Abbreviations: BMI, body mass index; PEFR, peak expiratory flow rate.

Table 2 Pearson's correlation coefficient between parameters and their $p$-values

\begin{tabular}{|l|l|l|l|l|l|l|}
\hline \multirow{2}{*}{ Parameter } & \multicolumn{2}{|c|}{ Combined } & \multicolumn{2}{c|}{ Male } & \multicolumn{2}{c|}{ Female } \\
\cline { 2 - 8 } & Correlation & $p$-Value & Correlation & $p$-Value & Correlation & $p$-Value \\
\hline WC and PEFR & 0.22 & 0.09 & -0.43 & 0.10 & 0.06 & 0.69 \\
\hline HC and PEFR & -0.004 & 0.97 & -0.22 & 0.40 & 0.06 & 0.70 \\
\hline WHR and PEFR & 0.37 & $<0.01$ & -0.48 & 0.06 & 0.04 & 0.78 \\
\hline
\end{tabular}

Abbreviations: HC, hip circumference; WC, waist circumference; WHR, waist-to-hip ratio; PEFR, peak expiratory flow rate. 
obesity markers was found in females. No correlation was demonstrated between PEFR and HC in the participants of either sex.

\section{Discussion}

The lifestyle and dietary habits in the urban areas of India have led to a high prevalence rate of obesity in the college-going young adults. ${ }^{8}$ It is important to find easy and readily available objective measures that can help to assess and regularly monitor the lung function and obesity parameters in an effective manner.

In this study, when combined data for both the genders were analyzed, a correlation was found among the three anthropometric measures, WC, HC, and WHR, which may act as obesity biomarkers, with PEFR. It was observed that both WC and WHR independently demonstrate an overall positive correlation with PEFR. However, this correlation was not statistically significant $(p>0.05)$ in both the cases. Several authors have suggested that a difference in body fat pattern exists in males and females and may have physiological implications. This probably might have led to the result being not significant statistically when data of males and females were studied together. However, a tendency toward a negative correlation between PEFR and WC $(r=-0.43)$ and between PEFR and WHR ( $r=-0.48)$ was observed in males, but it was not statistically significant. Also, no significant correlation of PEFR with any of the obesity markers was found in females. No correlation was revealed between PEFR and HC in the participants of either sex. A body fat accumulation pattern is proposed by several authors: for women, the fat is deposited around the hip region, whereas in men, the adiposity is observed in the abdominal area. As a consequence, perhaps, the effect of WC on pulmonary function in men tends to be larger than that in women. ${ }^{9,10}$ These scientists also suggested that abdominal fat may interfere in pulmonary mechanics, causing restrictions during breathing, potentially reducing respiratory volumes such as $\mathrm{FEV}_{1}$ (forced expiratory volume in 1 second) and FVC (forced vital capacity). This mechanical effect is more evident if central obesity is considered instead of overall obesity or peripheral fat. The excess of fat in the abdominal and thoracic region may lead to a decrease in the compliance and resistance of the respiratory system, increasing the energy demands of breathing., ${ }^{9,10}$

It was pointed out by Rochester and Enson that PEFR mainly depends upon height, chest size, and physical fitness of the individuals, which are presumed to be higher in males than in females. ${ }^{11}$ A study conducted by Saxena et al examined the effects of adiposity on PEFR in males and found that this parameter is negatively associated with obesity markers, as measured by BMI, WC, and WHR, after the effects of variation in age and height were removed. On multivariate analysis, WHR was found to be the most important parameter that showed a significant negative association with PEFR, whereas age, height, BMI, and WC were not. ${ }^{12}$ In a subsequent study, a positive correlation of PEFR with height and weight, as well as BMI was observed but was not found to be statistically significant. ${ }^{13}$ Collins et al also reported a lower $\mathrm{FEV}_{1}$ in participants with higher WHR even without adjustment for age, stature, and relative obesity. ${ }^{14}$ This study concluded that adiposity measured as WHR affects the PEFR in males in the age group of 20 to 40 years and especially when the age is approaching the higher side. These authors also suggested that WHR, a measure of central pattern of fat distribution, was associated with lower values for PEFR in young male adults after adjusting for BMI. ${ }^{14}$ The findings of the aforementioned studies also suggest that the pattern of body fat distribution has an independent effect on PEFR. ${ }^{12-14}$ In this study also, WC has shown a tendency of negative correlation with PEFR in males; however, no statistical correlation was found in females. The android and gynoid fat patterns found in males and females, respectively, may thus have their own physiological implications. Some recent studies have also suggested that WC is a better marker for mortality than BMI, as the BMI may get affected due to loss of lean muscle mass or that with advancing age. ${ }^{15}$ Chen et al investigated the effect of WC on PFT in three groups of people: normal weight, overweight, and obese. ${ }^{16}$ They found that there was a negatively significant relationship of WC with FEV1 as well as of WC with FVC. They also found that a $1-\mathrm{cm}$ increase in WC was associated with a $13-\mathrm{mL}$ reduction in FVC and 11-mL reduction in FEV1. All the aforementioned studies support the hypothesis that an excess fat in the abdominal and thoracic regions may limit movements of the diaphragm and decrease compliance of the respiratory system.

The physiology of obesity affecting the lung functions has been discussed in a landmark study where it has been shown that adipose tissue is metabolically active and has proinflammatory effects because it secretes a range of substances such as adipose-derived hormones called adipokines such as leptin, resistin, and adiponectin. The receptors for these hormones are widely distributed throughout the body, including the lungs. Leptin and resistin are increased in obesity and have proinflammatory effects, including activation of nuclear factor-kB and upregulation of tumor necrosis factor- $\alpha$. These changes lead to enhancement of neutrophilic airway inflammation in the lungs and cause deterioration in its function. In contrast to leptin and resistin, adiponectin is an anti-inflammatory adipokine that balances the activities of pro- and anti-inflammatory cytokines. It has also been suggested that adipose tissue derived hormones may play an important role in the pathophysiology of asthma in obese patients and can be therapeutically manipulated for its management. ${ }^{17}$

Normally, the decrease in lung function can be regularly monitored through PEFR, which can be measured with the use of a portable device in a cost-effective manner.

\section{Conclusion}

The lung function parameter PEFR appears to be adversely affected with an increase in WC and WHR in males but not in females. However, HC, another anthropometric measure, does not seem to affect the PEFR in the participants of either sex. 


\section{Conflict of Interest}

None declared.

\section{References}

1 World Health Organization, Obesity: Preventing and Managing the Global Epidemic. Geneva: World Health Organization; 1998

2 Dalton M, Cameron AJ, Zimmet PZ, et al; AusDiab Steering Committee. Waist circumference, waist-hip ratio and body mass index and their correlation with cardiovascular disease risk factors in Australian adults. J Intern Med 2003;254(6):555-563

3 Ashwell M, Mayhew L, Richardson J, Rickayzen B. Waist-toheight ratio is more predictive of years of life lost than body mass index. PLoS One 2014;9(9):e103483

4 Niyonsenga T, Carroll SJ, Coffee NT, Taylor AW, Daniel M. Are changes in depressive symptoms, general health and residential area socio-economic status associated with trajectories of waist circumference and body mass index? PLoS One 2020;15(1):e0227029

5 DeVrieze BW, Giwa AO, Peak Flow Rate Measurement. Treasure Island, FL: StatPearls; 2019

6 Neuspiel DR, Courtlandt CD, Windle ML. Peak Expiratory Flow Rate Measurement [Internet]. Available at: https://emedicine. medscape.com/article/1413347-print. Accessed March 27, 2020

7 Shenoy JP, Shivakumar J, Kalpana B, Bhat SK. Impact of adiposity markers on Peak expiratory flow rate in young adult South Indian females. Muller J Med Sci Res 2014;5:121-124

8 Ahirwar R, Mondal PR. Prevalence of obesity in India: a systematic review. Diabetes Metab Syndr 2019;13(1):318-321
9 Wehrmeister FC, Menezes AMB, Muniz LC, Martínez-Mesa J, Domingues MR, Horta BL. Waist circumference and pulmonary function: a systematic review and meta-analysis. Syst Rev 2012;1:55

10 Saraswathi I, Christy A, Saravanan A, Sembulingam P. Correlation of obesity indices with peak expiratory flow rate in males and females. IOSR Journal of Pharmacy 2014;4(2):21-27

11 Rochester DF, Enson Y. Current concepts in the pathogenesis of the obesity-hypoventilation syndrome. Mechanical and circulatory factors. Am J Med 1974;57(3):402-420

12 Saxena Y, Purwar B, Upmanyu R. Adiposity: determinant of peak expiratory flow rate in young Indian adults male. Indian J Chest Dis Allied Sci 2011;53(1):29-33

13 Gupta S, Rai RH. To determine the correlation of BMI, height and weight with peak expiratory flow rate in young adults. Int J Sci Res Rev 2019;8(8):163-169

14 Collins LC, Hoberty PD, Walker JF, Fletcher EC, Peiris AN. The effect of body fat distribution on pulmonary function tests. Chest 1995;107(5):1298-1302

15 Kim YH, Kim SM, Han KD, et al. Waist circumference and allcause mortality independent of body mass index in Korean population from the National Health Insurance Health Checkup 2009-2015. J Clin Med 2019;8(1):72

16 Chen Y, Rennie D, Cormier YF, Dosman J. Waist circumference is associated with pulmonary function in normal-weight, overweight, and obese subjects. Am J Clin Nutr 2007;85(1):35-39

17 Alipour B, Hosseini SZ, Sharifi A, Ansarin K. Influence of anthropometric measurements in lung function in patients with asthma. Int J Prev Med 2015;6:50 